\title{
La vie au cœur de la phénoménologie française
}

\section{Camille Riquier}

\section{OpenEdition}

\section{Journals}

Édition électronique

URL : http://journals.openedition.org/alter/856

DOI : $10.4000 /$ alter.856

ISSN : 2558-7927

\section{Éditeur :}

Association ALTER, Archives Husserl (CNRS-UMR 8547)

\section{Édition imprimée}

Date de publication : 1 novembre 2013

Pagination : 223-237

ISBN : 978-2-95-223749-9

ISSN : 1249-8947

\section{Référence électronique}

Camille Riquier, «La vie au cœur de la phénoménologie française », Alter [En ligne], 21 | 2013, mis en ligne le 01 juin 2019, consulté le 06 juillet 2019. URL : http://journals.openedition.org/alter/856 ; DOI : 10.4000/alter.856 


\section{LA VIE AU COEUR DE LA PHÉNOMÉNOLOGIE FRANÇAISE}

Camille Riquier

Que la vie, comme question, ait fini par occuper une place aussi privilégiée au sein de la philosophie française doit d'abord surprendre, s'il faut cumuler les attaques répétées qu'on a portées contre elle, voire les interdits qui ont toujours pesé sur elle. Tout se passe curieusement comme si à l'endroit où son interrogation était la plus menacée, toujours suspecte d'invoquer une signification vague et trompeuse, la notion de vie avait trouvé de quoi se renforcer, en se nourrissant et en se relevant toujours plus forte des objections mêmes qu'on lui avait adressées depuis Descartes. Présent d'abord de façon souterraine, le débat autour de la vie s'est affirmé crucial par la suite, aujourd'hui même, au point de pouvoir définir pour F. Worms le "moment » présent, par les multiples champs d'études qu'il investit comme par les nombreuses inquiétudes dont il se fait l'écho: «Le problème de la vie n'est plus un problème local, un problème parmi $\mathrm{d}^{\prime}$ autres, mais [...] l'on pourrait bien assister aujourd'hui à l'extension du problème ou du modèle du vivant à tous les domaines du savoir et de la pratique ${ }^{1}$. À l'ère des biotechnologies, des nanotechnologies, et des problèmes politiques liés à l'écologie, la vie subit des transformations telles qu'interroger sa nature est devenu une urgence. Et non sans quelque illusion rétrospective, elle redevient, "à l'heure de sa redécouverte ", le fil invisible qui a traversé la philosophie en France au $X X^{\mathrm{e}}$ siècle, et semble pouvoir expliquer pourquoi devant la «montée en puissance des modèles analytiques en philosophie »

\footnotetext{
${ }^{1}$ F. Worms, La Philosophie en France au 20e siècle, Paris, Gallimard, 2009, p. 562.
} 
s'était associée à son refoulement, comme l'a fait remarquer G. Le Blanc, « une éclipse de la philosophie française contemporaine $»^{2}$.

\section{1) Esquisse d'un tableau vivant de la phénoménologie française}

Il faut toujours commencer par rappeler que la vie est l'une des plus vieilles notions (Zoé/Bios), et qu'elle est pourtant, comme notion, sans passé. C'est qu'elle fut chaque fois privée des définitions clairement arrêtées qui en eussent autorisé la recollection au sein d'une histoire. A-t-on voulu écrire néanmoins son histoire? Ce n'était, de l'aveu même de ceux qui s'y risquaient, qu'en ramenant à la surface sa signification implicite et comme enfouie dans les textes de biologie et de physiologie, lesquels n'avaient jamais eu la vie pour objet $^{3}$. C'est dire que la vie telle qu'elle rejaillit dans la philosophie française au $X X^{\mathrm{e}}$ siècle est très loin d'être la dernière étape d'un parcours tâtonnant et toujours en progrès; elle est plutôt comme le retour du refoulé qui a fini par forcer le barrage qui l'avait exclu hors du champ rigoureux de la philosophie, en infiltrant ici ou là, de manière différenciée, tous ses nombreux domaines.

Parmi ces domaines, il faut compter la phénoménologie française et la compter à maints égards comme la plus représentative de cette tendance qui dut conduire la philosophie à s'attacher chaque fois plus à la vie, qu'elle découvre rétrospectivement avoir été le point aveugle de ses recherches. Car avant d'y venir, il y eut des obstacles à surmonter. En France, le premier obstacle fut le cartésianisme; le second fut Heidegger. L'ontologie classique, issue de Descartes, avait séparé la pensée et l'étendue et par là même dissout la vie. Pensant trop ou trop peu, elle se rendait incapable de tenir le milieu et de la saisir dans sa spécificité. C'était le vitalisme qui était rejeté en biologie, méprisé en philosophie. Plus tard, l'ontologie phénoménologique, assumée par Heidegger, disqualifiait à nouveau la vie, cette fois au profit de l'existence, faute de posséder pour elle un concept ontologiquement déterminé. Pouvait-on espérer plus d'elle que de l'ontologie cartésienne? Il était alors demandé d'attendre que l'ana-

\footnotetext{
2 G. Le Blanc, Canguilhem et la vie humaine, Paris, PUF, 2002, «Quadrige », 2010, Avant-propos à la présente édition, "Qu'est-ce qu'une philosophie de la vie aujourd'hui ? », p. 7. Voir également, F. Worms, "La philosophie de la vie en France au XXe siècle» (in Philosophie, numéro consacré à la Philosophie française contemporaine, printemps 2011).

${ }^{3}$ A. Pichot, Histoire de la notion de vie, Paris, TEL, « Gallimard », 1993.
} 
lytique du Dasein fût solidement établie pour éclairer en retour le mode d'être de la vie, et offrir ainsi une base ontologique suffisante à la constitution d'une phénoménologie qui lui fût ajustée.

Mais le détour exigé par Heidegger devait se prolonger sans fin et le condamner à manquer une nouvelle fois la vie dans son mode d'être propre. La méthode phénoménologique requise obligeait en effet à l'atteindre, elle qui n'est pas un objet (Vorhanden), " par la voie d'une privation réductive », " par voie d'interprétation privative » c'est-à-dire indirectement à partir du mode d'être du Dasein qu'elle n'était pas davantage. Bref, loin d'apporter le préalable nécessaire qu'on cherchait avant d'entreprendre une phénoménologie de la vie, l'ontologie fondamentale de Heidegger ne pouvait s'accomplir que sur fond de son impossibilité et d'une occultation foncière de la vie. C'est ce que suggère Didier Franck dans une excellente formule : « La disparition de la chair, du corps, est le prix phénoménologique de l'apparition de l'être $»^{4}$. On découvrait ainsi que la phénoménologie de l'être avait eu un coût réel et que son prix fut, une seconde fois, de sacrifier la vie dans son essence - à charge pour la phénoménologie qui lui succéda de savoir si les gains obtenus étaient plus grands ou moindres que les pertes consenties. Libre à la phénoménologie française encore naissante de revenir plus tard sur le point obstrué, d'y buter, et à la fin, une fois le passage forcé, d'y trouver le lieu de son déploiement.

Indéniablement, un tel discrédit jeté sur la vie avait frappé de mutisme la première vague de la phénoménologie française (Sartre, Merleau-Ponty) concernant son statut, autant par intimidation qu'en vertu d'une réelle difficulté à en saisir la nature à partir de la conceptualité héritée (conscience/monde). Il suffit de voir comment la génération suivante commença à y revenir, d'abord timidement, puis résolument, à mesure qu'elle s'éloignait de ses premiers maîtres et que resurgissait la notion de la vie comme son impensé le plus fondamental. Ce n'est que tardivement que Ricœur, à son tour, déplace l'axe de sa réflexion de la conscience vers la vie, ainsi qu'il en apporte lui-même le témoignage en 1995: «Je suis en ce moment en réflexion sur le thème de la vie, que j'avais toujours fui ; à la suite du premier Husserl, je me méfiais beaucoup de la Lebensphilosophie, de l'idée d'une philosophie de la vie $»^{5}$. Surmontant ses premières réticences, Ricœur peut alors étayer son refus d'accorder à l'être-pour-la-

${ }^{4}$ D. Franck, «L'être et le vivant », Dramatique des phénomènes, Paris, PUF, 2001, p. 55.

${ }^{5}$ P. Ricœur, La Critique et la conviction, Entretien avec F. Azouvi et M. de Launay, Paris, Hachette, 1995, p. 143. 
mort le même privilège que lui avait donné Heidegger, en entendant autrement la possibilité d'être un tout, par la réactivation temporalisante du concept diltheyien de « cohésion de la vie $»^{6}$ (Zusammenhang des Lebens). Soi-même comme un autre lui permet ensuite de s'interroger sur un tel soubassement vital, qui donne sa cohésion au soi par la mémoire qui le constitue, et que traverse le premier niveau éthique comme désir de vivre bien. En 2006, Derrida éclaire son propre parcours au détour d'une parenthèse qui révèle que la question de la vie fut un de ses fils rouges tout au long de ses recherches depuis La Voix et la phénomène (1967) : "L'analytique commence bien par un "je suis" dont l'examen ontologique est repris là où Descartes se serait arrêté, mais par un "je suis" qui, pour Heidegger comme pour Descartes, n'est pas d'abord un "je suis vivant" ou "je respire". Au cœur de toutes ces difficultés, il y a toujours l'impensé d'une pensée de la vie (c'est par là, par la question de la vie et du "présent vivant", de l'autobiographie de l'ego dans son présent vivant, que ma lecture déconstructrice de Husserl a commencé, et en vérité tout ce qui a pu $\mathrm{s}^{\prime}$ ensuivre) $»^{7}$. Derrida n'aurait pas tant critiqué le «présent vivant » husserlien qu'il aurait voulu décomposer la notion mixte de "présent vivant» et ainsi libérer la vie elle-même de l'horizon qui lui fut toujours imposé par une métaphysique de la présence. C'est à une telle relecture de l'œuvre derridienne que F. Worms nous invite, pour qui il n'y a de déconstruction que parce qu'en son fond la vie est là comme l'indéconstructible. Si Derrida rejette la vie comme présence, c'est pour accéder à « une autre idée de la vie » et à "une autre idée de la justice $»^{8}$. Enfin, une phénoménologie de la donation a-t-elle rendu Jean-Luc Marion si étranger à de telles questions, dont le parcours le plaça toujours à l'écart (des métaphysiques) de la vie ? Si l'auteur ne veut voir dans la vie que la métaphore, à peine voilée, de notre ignorance, il faut noter cependant qu'il la retrouve au détour d'une description phénoménologique de la naissance, au sein de laquelle semblent se cristalliser les traits les plus irréductibles de la donation. N'est-elle pas un don qui la situe d'emblée au-delà de tout objet, audelà de l'être lui-même? "La vie elle-même n'est pas quelque chose, encore moins un objet: on peut même douter que simplement elle soit. Rien n'est donné : la vie n'est rien, n'est pas ». "En elle, écrit-il encore, le don se réduit parfaitement à la donation, à ce rien qui

\footnotetext{
${ }^{6}$ P. Ricœur, Temps et récit (1985), Paris, Seuil, « Essais », 1991, vol. III, p. 132.

7 J. Derrida, L'animal que donc je suis, Paris, Galilée, 2005, p. 153.

${ }^{8}$ F. Worms, La Philosophie en France au XXe siècle, op. cit., p. 495.
} 
arrache tout au néant ${ }^{9}$. Ne pourrait-on pas alors élever la Vie au rang d'un paradigme où mieux qu'ailleurs peut se penser le phénomène de la donation? N'est-elle pas par excellence le don pur, puisqu'elle se déploie dans sa propre immanence tout en permettant de conclure à sa transcendance résolue par l'absence patente de son donateur?

L'intérêt porté à la vie fut ainsi acquis progressivement par la phénoménologie française. Et après avoir surmonté la méfiance qu'elle inspirait, on a pu redécouvrir sa présence jusque dans les textes des pères fondateurs de la phénoménologie, où le vocabulaire de la vie refluait parfois dans les formules de façon ininterrogée (Erlebnis, lebendige Gegenwart, etc.). Outre le dernier Husserl qui s'était penché sur la question du "monde de la vie»(Lebenswelt), la lente exhumation des inédits de Husserl atteste d'une attention croissante de sa part à la vie intentionnelle du sujet, sous-jacente à ses actes de constitution du monde, qui invitait à retourner "Husserl contre Husserl » selon la formule de A. Montavont. Car en s'enquérant d'une passivité originaire, c'est bien l'œuvre de constitution ellemême dont on s'apercevait qu'elle butait sur la vie comme sur un «fond d'inconstituable inintelligible, plaçant le sujet en position dérivée $»^{10}$. De façon parallèle, $l^{\prime}$ attention des commentateurs se portait également sur la période de gestation qui avait précédé la rédaction d'Être et temps, dans laquelle Heidegger formait encore le projet d'une herméneutique de la facticité, où la vie constituait le phénomène central ${ }^{11}$. Plus récemment, Jean Greisch traduisait enfin les cours déterminants de 1918 à 1921 qu'il regroupait sous le titre d'une Phénoménologie de la vie religieuse ${ }^{12}$.

Saisi par une telle convergence d'intérêts au sein des recherches en cours, Bruce Bégout avait déjà pu discerner «trois approches possibles d'une phénoménologie de la vie » dans un article récapitulatif très précieux. La première d'entre elles, de nature impressive, consisterait à aborder la vie par le sentiment interne; elle prendrait le parti que la vie se manifeste non à l'extérieur comme un principe de formation des êtres, mais dans le vécu lui-même (Scheler, Henry).

\footnotetext{
9 J. L. Marion, Certitudes négatives, Paris, Grasset, 2010, p. 298, 168. Il faut également citer la phénoménologie de la vie que $\mathrm{Cl}$. Romano découvre dans l'œuvre de Faulkner et à laquelle il rend la parole dans Le Chant de la vie. Phénoménologie de Faulkner, Paris, Gallimard, 2005.

10 A. Montavont, De la passivité dans la phénoménologie de Husserl, Paris, PUF, 1999, p. 273, 275.

${ }_{11}$ Cf. Heidegger 1919-1929, De l'herméneutique de la facticité à la métaphysique du Dasein, J. F. Courtine, J.-F. Marquet (dir.), Paris, Vrin, 1996.

${ }^{12}$ M. Heidegger, Phénoménologie de la vie religieuse, Paris, Gallimard, trad. J. Greisch, 2012. Voir aussi de J. Greisch, L'Arbre de vie et l'Arbre du savoir : le chemin phénoménologique de l'herméneutique heideggérienne (1919-1923), Paris, CERF, 2000.
} 
L'affectivité serait alors mise au premier plan. Par opposition, la seconde approche, de nature expressive, consisterait à côtoyer la vie dans son expression concrète, telle qu'elle se manifeste dans le monde et à travers les nombreuses formes qu'elle revêt (Dilthey, Simmel, Husserl et le premier Heidegger). Enfin, si l'on accepte que les deux approches contraires jouent en fait sur l'équivocité du vécu (Erlebnis) et du vivre (Leben), il est dit de la dernière qu'elle chercherait à atteindre, dans un geste ontologique plus radical, la vie en et pour elle-même, cette fois prise « en deçà des phénomènes vivants singuliers » en tant qu'elle constitue "l'essence de tous les vivants, et non plus seulement le mode d'être de l'homme dans le monde ${ }^{13}$ (Jonas, Barbaras).

Par sa clarté même un tel tableau occulte pourtant le mouvement d'affranchissement des interdits et des obstacles conceptuels qui ont peu à peu permis d'orienter la phénoménologie vers le phénomène de la vie. Car les auteurs évoqués n'y ont pas porté la même considération, ni au même titre, encore moins l'ont-ils tous pris pour centre de leur réflexion, en lui conférant une égale dignité. L'erreur de Bégout vient d'abord et avant tout d'avoir brodé les différentes possibilités offertes à la phénoménologie qui était en train de se faire sur l'a priori corrélationnel de la conscience, d'emblée comprise comme intentionnelle. Autrement dit, il s'est reposé sur lui de façon implicite, de telle sorte qu'il a pu s'ériger en juge depuis la pensée du maître tout en prétendant simplement faire un état des lieux. En effet, en régime de réduction phénoménologique, l'a priori corrélationnel de la conscience est la relativité essentielle qui rapporte l'objet au sujet dans l'intentionnalité. L'approche impressive et l'approche expressive renverraient ainsi aux deux pôles de la relation (sujet/objet), tandis que l'approche ontologique, seule disqualifiée, se situerait audelà des phénomènes, en vertu d'un mouvement de transgression, qui l'exclurait de la phénoménologie proprement dite. L'ultime tentative ne serait alors qu'une tentation, où la métaphysique fait retour, à laquelle l'auteur invite à ne pas céder. Toutefois, en continuant à mettre en œuvre les concepts opératoires de la phénoménologie traditionnelle, la tripartition proposée par Bégout se contente de répercuter sur le plan réduit de la phénoménalité la dualité des termes saisie dans leur relation intentionnelle. Son but semble être à la fin de

\footnotetext{
${ }^{13} \mathrm{~B}$. Bégout, «Le phénomène de la vie. Trois approches possibles d'une phénoménologie de la vie », in J.-M. Vaysse (éd.), Vie, monde, individuation, Hildesheim, Olms, 2003 ; repris, dans une version modifiée, sous le titre «Le phénomène de la vie et la phénoménologie », in Le Phénomène et son ombre, Chatou, Les Éditions de la Transparence, 2008, p. 167, 166.
} 
délimiter le champ légitime d'exercice de la phénoménologie, en le séparant soigneusement d'une ultime approche qui cesserait d'être phénoménologique en ce qu'elle assumerait déjà une position métaphysique. Pourtant, une telle critique, sous couvert d'offrir une cartographie des phénoménologies de la vie, n'est possible qu'en s'appuyant sur la phénoménologie husserlienne, dont on suppose qu'elle contient en germe et dans ses marges les phénoménologies à venir qui voudraient la détrôner. Husserl continue ainsi à régner en maître, en se prémunissant par avance contre ceux-là qui voudraient le renverser. N'est-on pas dès lors contraint de contester une telle critique en rappelant que la classification proposée par Bégout provient déjà d'un langage dans lequel la vie s'est diffractée, jouant sur l'équivocité du vécu (Erlebnis) et du vivre (Leben), rabattue de fait des deux côtés de l'intérieur et de l'extérieur, du dedans et du dehors, du sujet et de l'objet? En ce sens, approche impressive et approche expressive ne peuvent surgir que de manière dérivée et complémentaire et laissent inévitablement derrière eux, toujours déjà perdu pour la philosophie, le phénomène originaire de la vie, ramassé dans l'unité de son sens.

\section{2) Réduction ou intentionnalité ? \\ Michel Henry ou Renaud Barbaras}

C'est dire que la radicalité du questionnement que l'on attend d'une phénoménologie de la vie invite à adopter la vie comme un centre pour la pensée, là où la pensée doit pouvoir rejoindre l'être luimême en son propre centre. Car si la phénoménologie ne peut y aboutir, c'est qu'elle doit commencer avec elle, et se penser comme le vrai commencement, lequel oblige à revenir en deçà de ce que Michel Henry appelle «la phénoménologie historique »14. Autrement dit, la vie n'a pu faire que retour dans la phénoménologie, comme elle a fait retour partout en philosophie, à savoir comme son refoulé primordial; elle ne s'est pas obtenue à partir des prémisses posées par Husserl, mais contre elles, par subversion et retournement de leur sens et signification. Bref, une phénoménologie de la vie n'accède à sa propre possibilité qu'en opérant le démantèlement des deux concepts fondamentaux de la phénoménologie qui, dans leur appariement et leur renvoi réglé l'un à l'autre, servaient le projet husserlien de

${ }^{14}$ M. Henry, Phénoménologie matérielle, Paris, PUF, 1990, p. 62, 66, entre autres exemples. 
constitution du sens du monde, et empêchaient ni plus ni moins qu'apparaisse le phénomène fondamental de la vie comme apparaître originel et inconstituable. C'est qu'en effet, toujours mobilisées l'une par l'autre, la réduction définit sa méthode et l'intentionnalité son objet. La réduction phénoménologique opère l'épochè du monde et libère le sens de celui-ci en révélant la structure intentionnelle de la conscience, jusqu'ici dissimulée dans l'attitude naturelle. Et par choc en retour, l'intentionnalité autorise la réduction à être positivement une reconduction du regard, d'un regard tel qu'il se déporte vers la conscience en laquelle le monde se constitue. Mais c'est bien là manquer l'unité de la vie, doublement, en se constituant d'emblée prisonnier du partage entre la conscience et le monde, oscillant dans le meilleur des cas entre une vie transcendantale et une vie empirique. Car en s'ajustant l'une à l'autre, réduction et intentionnalité sont, par leur parfait ajointement, cela même qui interdit de laisser passer l'essence de la vie comme celle de l'apparaître en tant que tel. D'un côté, la réduction met bien entre parenthèses l'apparaissant, mais de telle façon que par l'intentionnalité qu'elle révèle il continue de s'adosser à l'apparaître, qu'il faut alors toujours entendre comme l'apparaître de cet apparaissant. De l'autre côté, l'intentionnalité déploie certes l'apparaître lui-même, mais de telle façon qu'elle se déporte, en raison de la réduction qui s'y reporte, vers les termes qui apparaissent en lui. Conscience et monde renvoient l'un à l'autre, et dans les deux cas, c'est l'essence de la vie qui est manquée dans sa phénoménalité pure.

Il n'y a donc de phénoménologies véritables de la vie que radicales, qui les obligent à s'affranchir entièrement de la phénoménologie historiquement constituée. Ne convient-il pas de configurer alors autrement le tableau initial, en éliminant des différentes doctrines celles qui ne satisfont pas à cette exigence de radicalité ? Comment néanmoins s'affranchir de la phénoménologie historique tout en conservant pour elles le titre de phénoménologie? Comment la phénoménologie peut-elle en être encore une si elle cesse de mettre en œuvre ses concepts les plus fondamentaux, la réduction et l'intentionnalité ? Voilà l'originalité : elle ne le peut qu'en les désappareillant; elle ne le peut qu'en les faisant jouer l'un contre l'autre plutôt que l'un par l'autre, en cessant de mobiliser ensemble leur valeur opératoire. Ainsi compris, deux phénoménologies de la vie se font vis-à-vis, et il ne peut y en avoir que deux possibles, selon le trait subversif qu'on aura préféré souligner : celle de Michel Henry, qui s'appuie sur la réduction, radicalement, et celle de Renaud Barbaras, qui relève l'intentionnalité, radicalement. 
C'est à leur confrontation que ces deux phénoménologies de la vie invitent tant la radicalité de leur questionnement les situe en un milieu commun, où tout semble en même temps les opposer. En effet, elles s'accordent pour voir dans la Vie l'essence de la manifestation $\mathrm{du}$ monde; elles ont d'ailleurs un même projet: désenvelopper l'apparaître lui-même de ce qui apparaît en lui afin de le faire apparaître en tant que tel - comme « apparaître pur » dont le vrai nom est la Vie. Mais elles diffèrent par les moyens employés et visent en direction contraire, au point que les résultats auxquels elles aboutissent vont s'opposer frontalement. Pour Michel Henry, la Vie s'écarte résolument du monde, et s'éprouve dans un contenu phénoménologique radicalement autre que le monde qui se manifeste en elle (dualisme phénoménologique); pour Renaud Barbaras, elle est au contraire proximité au monde, comme sa doublure invisible, qui témoigne de son essence foncièrement transitive (" monisme phénoménologique $»^{15}$ ). Nul doute qu'en les mettant en débat il faille avec eux interroger le cœur même de la phénoménologie, puisqu'en avançant au premier plan cette Vie que la phénoménologie avait jusqu'ici présupposée seulement, il y va en chacune de la nature de la phénoménologie elle-même.

On remarquera d'abord qu'en France la question s'est posée, quoique toujours implicitement, de savoir ce que la phénoménologie devait garder comme "sa pierre de touche non négociable » ${ }^{16}$, si elle voulait continuer à s'en prévaloir. Tout s'est passé comme s'il avait fallu choisir où mettre l'accent, entre l'intentionnalité (Sartre, Merleau-Ponty, Ricœur) et la réduction (Levinas, Derrida, Marion), là même où Husserl les pensait dans leur juste équilibre, abandonnant dans tous les cas l'idéalisme transcendantal de celui-ci et le projet de constitution qui l'accompagnait. C'est ainsi que deux générations de phénoménologues français ont pu s'ignorer superbement pour n'avoir pas salué en Husserl la même «idée fondamentale » ${ }^{17}$. N'estce pas alors devant cette même alternative que Michel Henry et Renaud Barbaras ont naturellement été conduits? Plus encore, c'est en avançant le plus loin possible dans la voie choisie qu'ils se sont

\footnotetext{
${ }^{15}$ Ibid., p. 311.

${ }_{16}$ J.-L. Marion, De Surcroît, Paris, PUF, 2001, p. 54, qui choisit d'ailleurs son camp au point d'occulter qu'il puisse y en avoir d'autre.

17 J.-P. Sartre, «Une idée fondamentale de la phénoménologie de Husserl : 1'intentionnalité », in Situations philosophiques, Paris, Gallimard, TEL, 1990, p. 9. Nous ne voulons absolument pas dire par là qu'ici ou là l'autre opération est absente, mais qu'elle est profondément remaniée, voire altérée et pour ainsi dire méconnaissable comme telle, en raison des déplacements effectués par chacun des auteurs.
} 
obligés à choisir résolument, invités du même coup à se situer aux deux antipodes de la phénoménologie française. Chacun se devait de désentraver sa propre voie des complications où l'autre devinait sa propre issue. Retraçons donc les deux parcours sommairement en les suivant tour à tour. Nous espérons par là qu'ils s'éclaireront aussi l'un par l'autre d'être ainsi comparés.

La phénoménologie de la vie portée par Renaud Barbaras repose sur la possibilité de mettre en œuvre l'intentionnalité contre la réduction phénoménologique elle-même. Pour autant, il n'est pas question de retomber dans l'attitude naïve et naturelle où la conscience se laisse hypnotisée par ce qui en elle apparaît, mais d'interroger l'intentionnalité en tant que telle. Or, c'est cela que nous interdit de faire la réduction au moment où pourtant elle nous la dévoile, puisqu'elle ne nous arrache au donné qu'en nous reconduisant aussitôt vers le vécu de conscience comme vers un autre donné qu'elle suppose plus originaire. Là où la conscience se pense dans le monde, la réduction nous invite simplement à la penser à l'origine du monde qui, lui, se constitue en elle. Bref, elle intervertit l'ordre des termes, mais continue de recouvrir l'apparaître par l'apparaissant, aussi primordial soit-il. On le voit, ce qu'a longtemps cherché Renaud Barbaras était d'exhiber l'intentionnalité elle-même, de penser l'a priori corrélationnel qu'elle enveloppe sans se laisser enfermer dans les termes de la corrélation, avec l'espoir d'y saisir l'essence originaire de la Vie dans laquelle la conscience et le monde se déploient en vis-àvis. Puisant d'abord dans les ressources qu'offrait la critique bergsonienne de l'idée de néant pour une phénoménologie du désir ${ }^{18}$, c'est la mise en œuvre d'une "épochè sans réduction » telle qu'il la trouve dans la philosophie de Jan Patocka qui lui permet de mettre au jour l'apparaître tel qu'il ouvre la transcendance pure du monde, avant tout renvoi en lui à la positivité d'un apparaissant: «Dans la mesure où l'épochè doit englober la position de tout étant apparaissant, par conséquent aussi celui qui s'apparaît à lui-même dans l'immanence, à savoir le vécu, elle ne débouche pas sur une réduction, c'est-à-dire une reconduction à une région d'un type singulier, la région conscience. Il s'ensuit que le phénoménal ne doit plus être décrit comme ce qui est constitué par le sujet sur la base de vécus immanents, comme chez Husserl, mais bien plutôt comme cela qui, relatif à un sujet, demeure pourtant transcendant, puisque c'est en lui

${ }_{18}$ R. Barbaras, Le désir et la distance, Paris, Vrin, 1999, ch. III. 
que tout étant, en tant qu'il est, trouve sa possibilité » ${ }^{19}$. Loin qu'elle doive quitter le monde pour s'étreindre dans l'immanence pure, comme le réclame Michel Henry, « la vie de la vie consiste dans un faire voir $»^{20}$ et ne tend à rien $\mathrm{d}^{\prime}$ autre qu'à la visibilité du monde. Il s'agit d'habiter l'intentionnalité, non pas de s'en extraire, afin de saisir «les termes de la corrélation du point de vue même de leur relation $»^{21}$, c'est-à-dire dans le mouvement même qui porte vers l'apparaître du monde. Depuis ses travaux sur Merleau-Ponty, les ouvrages de Barbaras s'orientent vers un affranchissement progressif de l'opposition conscience/monde dans laquelle Merleau-Ponty luimême reconnaissait sa marche entravée. Fort désormais d'une épochè sans réduction, qui doit nous prémunir contre toute objectivation et toute subjectivation, son auteur peut assumer pleinement de s'installer dans la vie et la penser comme la relation elle-même avant que son unité originaire ne se brise et ne se partage en conscience et en monde, en Erleben et en Leben. Ressaisi au-delà d'un tel partage, le sens d'être originaire de la vie ne peut se comprendre que comme Désir'22.

Dévoyant lui aussi l'héritage husserlien, n'est-ce pas la possibilité exactement inverse qui était endossée par la phénoménologie de Michel Henry, en se proposant alors d'exacerber la réduction phénoménologique au point de l'exercer contre l'intentionnalité ? C'est en effet dans les Leçons pour une phénoménologie de la conscience du temps que Michel Henry a d'abord considéré que Husserl a frôlé le phénomène de la vie, à mesure qu'il était invité, en remontant jusqu'aux formes primordiales de la constitution, à admettre la matière impressionnelle comme un donné irréductible aux formes intentionnelles qui l'animent. C'est cette matière qui doit porter en elle l'essence de la vie et qui aurait dû contraindre Husserl à lui conférer l'être en son essence propre, s'éprouvant elle-même sans se défaire de soi dans l'éternel présent de la Vie. Ce qui l'en a pourtant empêché fut d'avoir rapporté cette hylè, tel un contenu aveugle, à la morphè intentionnelle qui seule accomplit à ses yeux l'œuvre de manifestation. La réduction phénoménologique, simple ou double, reste amarrée à l'intentionnalité dont elle dévoile les multiples prestations au sein de la conscience constituante. Ainsi, en analysant l'autotemporalisation du temps,

\footnotetext{
${ }^{19}$ R. Barbaras, Le mouvement de l'existence. Etudes sur la phénoménologie de Jan Patocka, Chatou, La Transparence, 2007, 4e étude, p. 61-62.

${ }^{20}$ R. Barbaras, Introduction à une phénoménologie de la vie, Paris, Vrin, 2008, p. 265.

${ }^{21}$ Idem, p. 80.

${ }^{22}$ Nous nous permettons de renvoyer à notre article, $\grave{A}$ la lisière $d u$ monde : la vie selon Barbaras, in Critique, Paris, Minuit, no 750, nov. 2009, p. 981-993.
} 
Husserl s'est bien risqué à descendre dans les profondeurs hylétiques de la conscience, mais la subjectivité absolue, dès lors qu'elle continue de se rapporter intentionnellement à elle-même dans la rétention, creuse en elle une déhiscence temporelle, un premier écart à soi qui ouvre toujours déjà l'espace ek-statique où la vie est engloutie, où commence à poindre les premières lueurs du monde. Le Présent Vivant duquel jaillit l'impression originaire est donc toujours déjà pris dans le procès d'autoconstitution du flux phénoménologique de la conscience dans lequel plus rien ne subsiste: " Dans le flux il n'y a pas de vie réelle, il n'y a pas de vie au présent - il n'y a pas de vie possible $»^{23}$. Michel Henry n'a pas d'autres moyens de poser à nouveaux frais la question de l'autorévélation de la subjectivité absolue, sinon en brisant le rapport d'implication qui attachait étroitement ensemble réduction et intentionnalité. Car la réduction husserlienne, comme réflexion sur notre conscience spontanée, implique la rétention, qui est encore une intentionnalité, de telle sorte qu'elle emporte toujours déjà dans la lumière de son voir, en l'y projetant, l'être proprement non-intentionnel de l'impression, qu'elle déréalise par là même en le privant de son propre mode de donation.

Certes la "phénoménologie non intentionnelle », déployée par Henry, ne disqualifie pas tant l'intentionnalité qu'elle ne la reconduit à un fondement plus ancien qu'elle ${ }^{24}$, mais elle ne conquiert pas moins l'apparaître pur, où l'intentionnalité se révèle à elle-même, qu'en cherchant à l'en soustraire de part en part. Elle exige donc de se situer en deçà de l'intentionnalité et de saisir la Vie dans sa pure immanence à soi avant qu'elle ne soit jetée au-dehors d'elle-même dans l'élément de la représentation. Il est alors réducteur de vouloir résumer l'entreprise de Michel Henry à la prise en charge du sentiment interne, comme y invite Bégout. Car elle consiste au contraire à récuser l'étroite complicité qui associe le sujet et l'objet dans une même essence, et à dénoncer tous les faux dualismes qu'elle engendre, y compris celui du sens interne et du sens externe, qui masquent le véritable dualisme phénoménologique. Parce que la Vie comme auto-affectivité s'oppose au monde comme s'opposent deux modes purs de l'apparaître, elle doit ainsi récupérer dans son immanence pure les modes originels selon lesquels la phénoménalité se phénoménalise, qui échappent essentiellement à l'intentionnalité parce qu'ils la fondent. Pour cette raison, Michel Henry a besoin d'opérer

${ }^{23}$ M. Henry, Phénoménologie matérielle, op. cit., p. 53.

${ }^{24} \mathrm{Cf}$. Cl. Serban, "Michel Henry et la question du fondement de l'intentionnalité », Bulletin d'Analyse Phénoménologique, vol. 6, 2010. 
une « réduction phénoménologique radicale » qui n’a plus rien à voir «avec la réduction phénoménologique pratiquée par Husserl » ${ }^{25}$. Réduction «pure » et « radicale», au point de se retourner en «contre-réduction $»^{26}$, elle va jusqu'à écarter l'intentionnalité rétentionnelle qui longe la conscience et la retient hors d'elle-même. Rien d'étonnant à ce que Michel Henry reprenne à son compte la maxime phénoménologique de Jean-Luc Marion, convaincu aussi bien qu'il peut la conduire plus loin que ne le fait ce dernier: «autant de réduction, autant de donation $»^{27}$. C'est qu'il s'agit de pousser la réduction « à son terme », jusqu'à ce que l'impression vivante, rendue à elle-même, soit comme énuclée, et délivrée de la gangue intentionnelle qui l'aliénait et l'empêchait d'être elle-même dans l'épreuve pathétique du vivre.

Pourquoi enfin cette double percée telle qu'elle permit de rejoindre le phénomène originaire de la vie, au sein du paysage que nous venons rapidement de retracer, s'est-elle toutefois faite en France plutôt qu'en Allemagne? L'œuvre décisive de Schopenhauer n'avaitelle pas ouvert «à la pensée moderne la voie de la vie ${ }^{28}$ ? C'est qu'il faudrait montrer que la phénoménologie historiquement constituée en Allemagne l'a autant rendue possible qu'elle l'a empêchée. Si d'ailleurs nous isolions le texte de Husserl, et que nous voulions expliquer ses héritiers français à partir de lui seul, par les nouvelles combinaisons de pensée qu'ils ont conquis dans ses marges, ainsi que nous l'avons fait en dissociant tel concept de tel autre porté à l'excès, nous n'aboutirions qu'à des abstractions spéculatives, aussi formelles que gratuites - et pour tout dire métaphysiques. Plus encore, à s'en tenir là, générée par l'écart introduit entre la réduction et l'intentionnalité, l'alternative proposée entre deux phénoménologies de la vie serait elle-même arbitraire. D'un point de vue husserlien, l'archiimpression (Henry) ou l'hyper-désir (Barbaras) sont des non-phénomènes et, partant, des possibilités vides auxquelles ne renvoie aucune expérience. Aussi est-il essentiel d'ajouter que l'une et l'autre ont dû prendre leur source dans une toute autre tradition de pensée, qui seule les autorisait à travailler en sous-main le texte husserlien - en y

\footnotetext{
${ }^{25} \mathrm{M}$. Henry, «Phénoménologie non intentionnelle : une tâche de la phénoménologie à venir », in De la phénoménologie, Paris, PUF, 2003, p. 118.

${ }^{26}$ Idem.

${ }^{27}$ J.-L. Marion, Réduction et donation. Recherches sur Husserl, Heidegger et la phénoménologie, Paris, PUF, 1990, p. 302 ; M. Henry, "Quatre principes de la phénoménologie », De la phénoménologie, tome I, op. cit., p. 77 sq.

${ }_{28}$ M. Henry, Généalogie de la psychanalyse, Paris, PUF, 1985, p. 247.
} 
faisant remonter une expérience primordiale qu'il ignorait, comme par infiltration. Cette tradition, plus méconnue, demeure ancrée dans « la vie intérieure et personnelle ${ }^{29}$ et se trouve représentée en France depuis Descartes par Pascal, Rousseau, Maine de Biran ou encore Bergson. La phénoménologie française a pu ignorer, négliger voire se méprendre sur l'un de ses maillons, c'est pourtant à cette longue chaîne que les phénoménologies de la vie se rattachent à nos yeux, qu'elles se sont proposées chacune de poursuivre de manière réglée et différenciée. Ce fut d'ailleurs la figure de Maine de Biran que Michel Henry et Renaud Barbaras d'abord privilégièrent. Et quand celui-ci est amené à s'expliquer avec la philosophie de celui-là sur le sens qu'il faut accorder à la vie, il ne lui reproche pas son manque d'orthodoxie husserlienne, il lui dispute l'interprétation qu'elle propose de l'effort « hyperorganique » biranien. Pour Michel Henry, il est le pouvoir qui coïncide avec lui-même, dans l'immanence d'une intériorité pure, en tant qu'il caractérise la Vie même ; pour Renaud Barbaras, il est au contraire le pouvoir de se mouvoir, qui, sans être rendu étranger à lui-même, est déjà arrachement, jeté dans l'élément de l'extériorité. Maine de Biran se serait-il reconnu dans l'une de ces deux définitions? Probablement non, mais peu importe. Comme le souligne Renaud Barbaras, il continue même de rabattre la vie sur le plan de l'organisme objectif, loin de l'identifier à l'être du mouvement ou même à l'immanence de la subjectivité. Mais, c'est qu'alors on doit reconnaître en germe dans le biranisme la voie pour trois acceptions différentes, au moins, de la vie, qui rend le dialogue possible entre les deux phénoménologues: pulsion, désir ou organisation ${ }^{30}$.

Pouvait-on remonter plus haut encore dans une archi-phénoménologie, qui pût attester le caractère expérientiel de la vie ainsi traduite? Ce fut alors pour l'un comme pour l'autre l'occasion de replonger jusque dans la doctrine-mère de Descartes, et de l'extraire du cartésianisme officiel en prenant pour levier une lecture déterminée du Cogito : d'un côté, l'âme dont on n'a aucune idée (« at certe videre videor »); de l'autre, le sentiment de l'union, lui aussi avant que l'idée qu'on en a ne le scinde en deux et ne le fige dans l'opposition conscience / monde. Décidément, en «terre " cartésienne,

\footnotetext{
${ }^{29}$ M. Henry, "Le concept d'âme a-t-il un sens ? », in De la phénoménologie, Paris, PUF, 2003, p. 23. Voir aussi notre article, «Henry, Bergson et la phénoménologie matérielle », in Michel Henry's Radical phenomenology, R. Kühn et J. Hatem (dir.), Studia Phaenomenologica, R. Kühn (éd.), 2009.

${ }^{30}$ R. Barbaras, "L'essence de la vie: Pulsion ou désir ? Sur Michel Henry », in La Vie lacunaire, Paris, Vrin, 2011, p. 35 sq.
} 
Husserl ne pouvait arriver que second, et ici et là c'est encore le Cogito cartésien, plus large et profond que le Cogito kantien ou husserlien, qui permît de travailler en sous-main les concepts opératoires de la phénoménologie et de laisser passer à travers eux une certaine expérience de la vie. C'est à partir de ce " cartésianisme des origines » qu'il a été et qu'il est encore possible d'étendre le champ de la phénoménologie, en intégrant en elle une expérience de la vie que la phénoménologie historique n'avait point connue, qu'elle avait même exclue a priori. C'est en revenant à la pensée cartésienne, comme nous voudrions pouvoir le faire ailleurs, qu'il peut également être montré la puissance et la subtilité des moyens que la phénoménologie offre pour penser la vie, qui pourtant résiste à la pensée tant elle « peut à peine être pensée $»^{31}$.

${ }^{31}$ M. Henry, «Qu'est-ce que cela que nous appelons la vie ? », dans De la phénoménologie, Paris, PUF, 2003, p. 41. 